\section{Studie bestätigt Wirksamkeit von Neurodoron ${ }^{\circledR}$}

Zwischen Dezember 2008 und August 2009 rekrutierten 43 Ärzte 300 Patienten mit nervöser Erschöpfung, deren Erkrankung seit mehr als 2 Jahren bestand. Bei 117 dieser Patienten wurde zudem Burnout diagnostiziert. Diese Patienten erhielten im Mittel über 46 Tage Neurodoron ${ }^{\circledR}$. Alle 39 dokumentierten Symptome wie Reizbarkeit, Kopfschmerzen und Schlafstörungen gingen deutlich zurück - in Summe gesehen um 58,4\%. Bei guter Verträglichkeit bestätigten rund $80 \%$ der Patienten und Ärzte eine gute bis sehr gute Wirksamkeit.

Erfahrungsberichte aus der ärztlichen Praxis weisen bereits seit Jahren auf eine gute Wirksamkeit von Neurodoron ${ }^{\circledR}$ bei stressbedingten Beschwerden hin. Mit der vorliegenden Beobachtungsstudie sollten weitere Erkenntnisse über die Wirksamkeit und Verträglichkeit von Neurodoron ${ }^{\circledR}$ in der ärztlichen Praxis gewonnen werden, insbesondere bei Patienten, die infolge von Stress unter nervöser Erschöpfung leiden. Bei dieser Untersuchung handelt es sich um eine offene, prospektive, multizentrische, nicht interventionelle Beobachtungsstudie zum therapeutischen Einsatz von Neurodoron ${ }^{\circledR}$ im zugelassenen Anwendungsbereich. Alle Entscheidungen hinsichtlich Patientenauswahl, Diagnostik und Therapie verblieben ausschließlich beim behandelnden Arzt. Pro Patient waren 2 Untersuchungen geplant - eine Aufnahme- sowie eine Abschlussuntersuchung nach $42( \pm 3)$ Tagen.

Bereits vor Beginn der Neurodoron ${ }^{\circledR}$-Behandlung erfolgte bei 62 Patienten $(20,7 \%)$ eine medikamentöse Therapie der nervösen Erschöpfung, am häufigsten mit «pflanzlichen Hypnotika/Sedativa» (15 Nennungen), gefolgt von «homöopathischen Hypnotika» und «pflanzlichen Antidepressiva» (je 12 Nennungen). Die Wirksamkeit der Vormedikation wurde von den Ärzten in 24,1\% aller Fälle mit «unbefriedigend» angegeben. Die Patienten vergaben diese Bewertung sogar für 34,5\% aller eingesetzten Präparate.

Von den 300 Patienten waren 234 (78\%) weiblichen und 66 (22\%) männlichen Geschlechts. Das durchschnittliche Alter betrug bei Behandlungsbeginn 50 Jahre. Mehr als 50\% der Patienten lagen im Bereich von 41 bis 60 Jahren. $61 \%$ aller Patienten gaben an, berufstätig zu sein. Bei fast der Hälfte der im Berufsleben stehenden Patienten (47\%) beinhaltete die berufliche Tätigkeit außergewöhnliche Arbeitszeiten wie Nacht- und Wochenendarbeit. Im Mittel dauerte es $12 \pm 6,9$ Tage (Median: 14 Tage), bis die Patienten eine erste Wirkung feststellten. Die zusammenfassende Wirksamkeitsbeurteilung der Neurodoron ${ }^{\circledR}$-Therapie fiel in 84,3\% aller Fälle (Ärztebeurteilung) beziehungsweise $78,7 \%$ (Patientenbeurteilung) «sehr gut» oder «gut» aus.

Alle Patienten litten unter nervöser Erschöpfung, im Mittel seit mehr als 2 Jahren. Bei 117 Patienten (39\%) wurde zusätzlich ein BurnoutSyndrom diagnostiziert; die diesbezügliche Erstdiagnose lag im Mittel circa 1,5 Jahre zurück. Als verantwortliche Ursachen wurden von den Patienten in der Reihenfolge genannt: «Beruf/Schule», «Haushalt», «Kinder», «Pflege Angehöriger», «traumatisches Ereignis» und «andere Ursache». Bei fast der Hälfte der Patienten wurde mehr als eine Ursache angegeben. Die teilnehmenden Ärzte verordneten Neurodoron ${ }^{\circledR}$ der überwiegenden Mehrheit der Patienten (74\%) in der Standarddosierung (3- bis 4-mal 1 Tablette pro Tag). Bei 17,7\% (53) der Patienten wurde eine höhere Dosierung angewendet. Etwa zwei Drittel der Patienten beabsichtigen, die Neurodoron ${ }^{\circledR}$-Therapie über die Dauer der Beobachtungsstudie hinaus fortzusetzen.
Eine Wirksamkeit von Neurodoron ${ }^{\circledR}$ konnten 266 der 300 Patienten $(88,7 \%)$ feststellen und zeitlich bestimmen. 32 Patienten (10,7\%) gaben an, keine ausreichende Wirksamkeit verspürt zu haben; bei 2 Patienten fehlten diesbezügliche Angaben.

Folgende stressassoziierten Symptome ließen sich während der Anwendung von Neurodoron ${ }^{\circledR}$ lindern: Psychische Symptome wie Antriebsschwäche, depressive Verstimmung, Konzentrationsschwäche, Nervosität, Reizbarkeit und Ruhelosigkeit. Körperliche Symptome wie Blutdruckauffälligkeiten, Kopfschmerzen, Muskelverspannungen, Rückenbeschwerden und Schlafstörungen.

Die Verträglichkeit bewerteten $97,4 \%$ der Ärzte und 95,7\% der Patienten mit «sehr gut» oder «gut». Insgesamt würden 90,7\% der 300 Patienten Neurodoron ${ }^{\circledR}$ weiterempfehlen. 97,3\% der Ärzte würden das Präparat bei weiteren Patienten einsetzen.

Neurodoron ${ }^{\circledR}$ zeigte im Rahmen dieser Beobachtungsstudie eine sehr gute Wirksamkeit und Verträglichkeit bei der Behandlung von Patienten, die aufgrund von Stress unter nervöser Erschöpfung und Burnout leiden. Das insgesamt sehr positive Arzturteil zur Entwicklung der Symptomatik wurde durch die Patienten bestätigt. Eine spürbare Wirkung trat im Mittel nach 14 Tagen auf. Ärzte sollten ihre Patienten daher auf die Notwendigkeit einer längerfristigen Neurodoron ${ }^{\circledR}$-Einnahme vorbereiten.

\section{Quelle}

Rother C, Oexle J: Einsatz von Neurodoron ${ }^{\circledR}$ bei Patienten mit nervöser Erschöpfung aufgrund von Stress. Merkurstab 2010; 63:171-177.

Weitere Informationen bei

Weleda AG

Dr. Sibylle Fuchs

Möhlerstraße 3, 73525 Schwäbisch Gmünd

Tel.+ 4907171 919-0

www.weleda.de

\title{
Ticker+++ Ticker+++ Ticker+++ Ticker+++ Ticker+++ Ticker+++ Ticker+++
}

G. Pohl-Boskamp. Nicht nur Hals-NasenOhren-Ärzte, sondern auch die große Mehrzahl der Allgemeinmediziner, Praktiker und Internisten (API) empfehlen Sesamöl (GeloSitin ${ }^{\circledR}$ Nasenpflege) zur nachhaltigen Befeuchtung der trockenen Nasenschleimhaut. Das zeigte jetzt eine Marktuntersuchung der G. Pohl-Boskamp GmbH \& Co. KG vom Dezember 2009, an der sich rund 23100 Ärzte beteiligten.

Weitere Informationen bei

G. Pohl-Boskamp GmbH \& Co. KG

Stefan Titzrath

s.titzrath@pohl-boskamp.de
Harras Pharma Curarina. In einer klinischen Doppelblindstudie an der Universitätsklinik für Dermatologie, Karls-Universität Prag, zeigte sich, dass die 2-fach-Kombination aus der pflanzlichen Wirksubstanz Levomenol (alpha-Bisabolol) und der Natursubstanz Heparin eine höhere Wirksamkeit gegen Ekzeme und Juckreiz entwickelt als ein pflanzlicher Wirkstoff allein. Die Besonderheit dieser Wirkstoffkombination (Sensicutan ${ }^{\circledR}$ Creme) liegt in der gegenseitigen Verstärkung der beiden Wirkstoffe.

Weitere Informationen bei

Harras Pharma Curarina GmbH

Ruth Komorek

ruth.komorek@harraspharma.de
Cefak. In einer multizentrischen Anwendungsbeobachtung zeigten die Ergebnisse, dass Cefase $^{\circledR}$ bei Patienten mit onkologischem Hintergrund eine signifikante Verbesserung von Immunkompetenz, klinischen Symptomen und Lebensqualität erzielen. Die teilnehmenden Ärzte beurteilten die Wirksamkeit der Cefase ${ }^{\circledR}$-Therapie hinsichtlich des Selenspiegels/Selenmangels bei knapp $76 \%$ der 681 Patienten mit «sehr gut» oder mit «deutlicher Besserung".

Weitere Informationen bei Cefak KG, Cefasel-Service cefak@cefak.com 